\title{
RNF43 Inhibits Cancer Cell Proliferation and Could be a Potential Prognostic Factor for Human Gastric Carcinoma
}

\author{
Lei Niu Hong-Zhen Qin Hong-Qing Xi Bo Wei Shao-You Xia Lin Chen
}

Department of General Surgery, Chinese People's Liberation Army General Hospital, Beijing, China

\section{Key Words}

RNF43 • E3 ubiquitin ligases • Gastric carcinoma • Wnt signaling • Tumor suppressor

\begin{abstract}
Background/Aims: RNF43 is a member of transmembrane E3 ubiquitin ligases and plays important roles in tumor formation progression. In current study, we aimed to explore RNF43 expression and analyze its role in gastric carcinoma. Methods and Results: The level of RNF43 was detected in 77 cases of gastric carcinoma and matched normal tissues by real-time PCR, western blotting and immunohistochemistry. We found that the expression of RNF43 was significantly down-regulated in the gastric carcinoma tissues compared to the normal mucosae (all $\mathrm{P}<0.001$ ). In addition, RNF43 was significantly correlated with histological differentiation $(P=0.001)$, T-stage cancer $(P<0.001)$, depth of invasion $(P<0.001)$, metastasis of regional lymph nodes $(P<0.001)$, pTNM stage $(P<0.001)$ and survival $(P=0.021)$. We further explored the biological functions of RNF43 in gastric carcinoma cell lines. Both gain- and loss-function assays show that RNF43 could suppress cell proliferation while promotes cell apoptosis. Further, we found that RNF43 was positively correlated with p53 and cleaved-caspase 3 and negatively correlated with Ki67 and Lgr5. Concolusion: In conclusion, RNF43 might act as a tumor suppressor in gastric carcinoma and might be a potential indicator for the clinical assessment of gastric cancer prognosis.
\end{abstract}

Copyright $\odot 2015$ S. Karger AG, Basel

\section{Introduction}

As the fourth most common malignant tumor, the mortality rate of gastric cancer ranks second worldwide [1]. Currently, the poor prognoses of that gastric cancer are because this condition is normally diagnosed in the advanced stages. Furthermore, curative treatments, such as surgery, radiotherapy and chemotherapy, are not able to prevent

Lin Chen, Professor,

KARGER 125
Department of General Surgery, Chinese People's Liberation Army General Hospital, 28 Fuxing Road, Beijing 100853 (China)

Tel. 86-010-66938448, E-Mail chenlinpla@163.com 
recurrence and metastasis, which are the ultimately the leading causes of death in patients with gastric cancer. Tumor progression might be related to the alteration of genes and the over-activation of signal pathways that regulate abnormal cell renewal [2-5]. However, the exact mechanisms of gastric cancer are not yet fully understood. As a critical pathway in the regulation of proliferation, Wnt/ $\beta$-catenin signaling is considered to play critical roles in embryonic development, adult tissue homeostasis [6] and aberrant activation in tumorigenesis in cancers [7].

Mutations in the downstream components of the Wnt pathway often cause cancer incidents. However, most mutations of the downstream Wnt signaling pathway cannot be inhibited by the Wnt inhibitors that are currently being developed. Recently, Hao et al [8]. and Koo et al [9]. simultaneously reported the unique transmembrane E3 ubiquitin ligase RNF43, which suppresses the initiation of Wnt/ $\beta$-catenin and Wnt/PCP signaling by endogenous Wnt proteins, and this process is different from those of other negative regulators of Wnt signaling, such as APC and AXIN [10]. However, the expression level and function of RNF43 in gastric carcinoma have not been examined. Here, we found low levels of expression of RNF43 in gastric carcinoma tissues compared to adjacent normal gastric tissues. Our work further suggests that the over-expression of the RNF43 suppresses proliferation and induces apoptosis in gastric carcinoma cells. Moreover, the opposite results were achieved in the knockdown test. We conclude that RNF43 might also act as a tumor suppressor in gastric carcinoma.

\section{Materials and Methods}

Cell culture and quantitative reverse transcription polymerase chain reaction (qRT-PCR)

The human gastric carcinoma cell lines (BGC823, MGC-803, and SGC7901) and a human gastric epithelial cell line (GES-1) were obtained from the American Tissue Culture Collection (ATCC, Manassas, VA, USA) and were cultured in 1640 and DMEM media containing 10\% fetal bovine serum (FBS). Quantitative RT-PCR analyses were performed on an ABI7700 sequence detection system (Applied Biosystems) using SYBR Green PCR Master Mix (Applied Biosystems). The nucleotide sequences of specific primers for the selected genes were as follows: RNF43 forward primer: (5-AGCATGAGTGGTGGCCACCAG-3), reverse primer (5-ATCTCACACAGCCTGTTCAC-3); and glyceraldehyde 3-phosphate dehydrogenase (GAPDH) forward primer: (5-CCACCCATGGCAAAATTCCATGGCA-3), reverse primer: (5-TCTAGACGGCAGGTCAGGTCCACC-3). The relative values of the expressions of the transcripts were calculated using the $2^{-\triangle \triangle C}$ (T) $\operatorname{method}[11,12]$. The mRNA expression level of RNF43 was normalized to that of GAPDH. All experiments were performed in triplicate.

Gastric cancer tissue specimens

Paraffin-embedded tissue specimens of 77 primary gastric carcinomas and 77 samples of matched normal mucosal tissues adjacent to the carcinomas were obtained from the Department of Pathology of the Chinese People's Liberation Army (PLA) General Hospital (Beijing, China) from 2002 to 2007. An additional four pairs of tissue specimens were used for western blot examinations. Ethical approval for this study was not required by our institution because the experiments were not related to the patients' privacy, impairment, or treatment. Histological specimens from each patient were reviewed to confirm the diagnoses and clinic pathological parameters. Pathological TNM staging was evaluated according to the 2009 criteria of the International Union Against Cancer (UICC). All of the patients were followed up for survival analysis. The follow-up period was calculated from the date of surgery until October 31, 2012.

\section{Immunohistochemistry}

Immunohistochemical staining was performed on 3- to 4- $\mu \mathrm{m}$ sections of formalin-fixed, paraffinembedded tissues. The slides were dew axed in xylene and rehydrated. The slides were heated in $0.01 \mathrm{~mol} / \mathrm{L}$ citrate buffer ( $\mathrm{pH} 6.0$ ) in a microwave oven for $2 \mathrm{~min}$ and $30 \mathrm{~s}$ at $100^{\circ} \mathrm{C}$ for antigen retrieval. Next, the slides were incubated for $15 \mathrm{~min}$ with 3\% hydrogen peroxide to inhibit endogenous peroxidase activity. After a washing with phosphate-buffered saline (PBS) and blocking with $10 \%$ goat serum, the sections were treated 
with primary polyclonal rabbit antibody to human RNF43 (ab129401, Abcam, MA, USA) diluted 1:100 in blocking solution and incubated over-night at $4^{\circ} \mathrm{C}$. After washing with PBS, the sections were incubated for 30 min with biotinylated secondary antibody (polyperoxidase-anti-mouse/rabbit IgG; Zymed Laboratories, South San Francisco, CA, USA), and peroxidase reactivity was then visualized using a 3,30-diaminobenzidine (DAB) substrate kit (Zymed). Finally, the slides were counterstained with hematoxylin. The primary antibody was replaced by PBS as a negative control.

\section{Evaluation of immunohistochemistry}

Antigen expression was independently evaluated using a light microscope by two investigators who were blind to all clinical pathology data. When there were discordant cases between the two investigators, the slides were reassessed on a double-headed microscope to establish a final result. For the RNF43 assessment, the immunohistochemical stainings of cells were evaluated according to a score that combined an intensity of staining scale with the percentage of cells stained [13]. The intensity of staining was scored as follows: 0 , no staining; $1+$, weak staining; $2+$, moderate staining; and $3+$, intense staining. The percentage of stained cells was scored as follows: 0 , no staining of any cells; $1+$, positive staining in $<10 \%$ of the cells; $2+$, positive staining covering $10-50 \%$ of the cells; and $3+$, positive staining of $>50 \%$ of the cells. The final score was determined as the combination of these scores. Scores (extent + intensity) of $\leq 1$ was considered negative, and scores between 2 and 6 were considered positive [14, 15].

\section{Protein extraction and western blotting}

Protein extraction and western blotting were performed as previously described [16]. Tissue and cell lysates were prepared with lysis buffer. The protein content of the supernatant was measured using a standard method. The protein samples were loaded at $50 \mu \mathrm{g} / \mathrm{lane}$ in $12 \%$ sodium dodecylsulfate (SDS), separated by polyacrylamide gel electrophoresis (PAGE), and transferred to a nitrocellulose membrane (Amersham Biosciences, Piscataway, NJ, USA). The membranes were blocked with 5\% non-fat milk in TBST (50 mmol/L Tris-HCl [pH 7.6], $150 \mathrm{mmol} / \mathrm{L} \mathrm{NaCl,} \mathrm{0.1 \%} \mathrm{Tween} \mathrm{20)} \mathrm{for} 1 \mathrm{~h}$ at room temperature and then incubated with primary antibody (anti-RNF43, Abcam, USA, 1:200; GADPH, 1:1000, Santa Cruz Biotechnology) in blocking buffer at $4^{\circ} \mathrm{C}$ overnight. After washing with TBST, the membranes were incubated with horseradish peroxidase-coupled goat anti-rabbit secondary antibody (1:10000; Santa Cruz Bio-technology) for $2 \mathrm{~h}$ at room temperature. Enhanced chemiluminescence was used for detection. The $\beta$-actin bands were used as the loading controls. The protein quantity was analyzed with Quantity-One v4.4 software (Bio-Rad, Hercules, CA, USA). The target protein expression was evaluated as the relative intensity ratio of the target protein to the loading control.

\section{Construction of recombinant adenovirus encoding RNF43 and lentivirus harboring RNF43 siRNA}

For recombinant adenovirus construction, the RNF43 cDNA (Ad-RNF43) and the green fluorescence protein gene (Ad-GFP; control) cloned by PCR was inserted into pDC315-EGFP vector (purchased from Hanbio Co. Ltd, Shanghai, China) respectively, under the control of the mouse cytomegalovirus (CMV) promoter. The pDC315-X and pBHGlox E1,3Cre were co-transfected into HEK293 cells to generate the recombinant adenoviruses. Ad- RNF43 and Ad-GFP were propagated in HEK293 cells. The Propagated recombinant adenoviruses in the HEK293 cells were purified and the titer of virus was measured by plaque assays The stock solutions of Ad- RNF43 and Ad-GFP were $1 \times 10^{10}$ plaque formation unit (PFU)/ml, respectively.

For lentivirus construction, oligonucleotides with the following targeting sequences were used for the cloning of small hairpin RNA (shRNA)-encoding sequences in hU6-MCS-PGK-EGFP lentiviral RNAi vector (Hanbio, Shanghai, China): RNF43, 5'- GCAGGAGCTACGGGTCATTTC-3'. The recombinant lentivirus with shRNA-RNF43 was produced by co-transfection of 293T cells with plasmids PSPAX2 and PMD2G. Lentiviruscontaining supernatant were harvested $48 \mathrm{~h}$ after transfection and filtered through $0.22 \mu \mathrm{m}$ cellulose acetate filters (Millipore, USA). Recombinant lentiviruses were concentrated by ultracentrifugation $(2 \mathrm{~h}$ at $50,000 \times \mathrm{g})$.

Cell apoptosis assay and cell proliferation assay

Cell apoptosis was measured at $72 \mathrm{~h}$ after infection using flow cytometry with the Annexin V-FITC Apoptosis Detection kit (BD bioscience) according to the manufacturer's instructions. Briefly, MGC803, BGC823 and GES-1 cells were digested with $0.25 \%(\mathrm{w} / \mathrm{v})$ trypsin and resuspended in $100 \mu \mathrm{L} 1 \times$ binding 
buffer to a final concentration of $1 \times 10^{5}$ cells. Five microliters of Annexin V-FITC were added to $100 \mu \mathrm{L}$ of the cell suspension. After a 20-min incubation at room temperature in the dark, the cell suspension was centrifuged at $1,000 \mathrm{~g}$ for $5 \mathrm{~min}$ and then resuspended in $400 \mu \mathrm{L}$ binding buffer and $3 \mu \mathrm{L}$ PI on ice in the dark. Subsequently, the number of stained cells was assessed with a flow cytometer (BD FACS AriaII, NJ, USA).

The Cell Counting Kit-8 (CCK-8) (Dojindo, Kumamoto, Japan) was used to measure the effects of RNF43 on tumor cell proliferation. The cells were plated at a density of 5,000 cells per well in 96-well plates in the presence of DMEM. The CCK-8 assays were performed at 0, 24, 48 and $72 \mathrm{~h}$ after infection. Ten micro liters of the cell proliferation reagent WST-8 were added to each well, and the cells were incubated for $1 \mathrm{~h}$ at 37 ${ }^{\circ} \mathrm{C}$. Viable cell counts were estimated by measuring the optical density at $450 \mathrm{~nm}$. The amount of the dye generated by the dehydrogenase reaction in the cells is directly proportional to the number of living cells.

\section{Statistical analyses}

SPSS V.17.0 (SPSS, Chicago, IL, USA) was used for the statistical analysis. The data regarding the relative expressions of RNF43 mRNA and protein are presented as the means \pm the SDs. Paired-samples-t-tests were used to assess differences in the relative expressions of RNF43 mRNA and RNF43 protein between the gastric cancer cell lines and the normal mucosa cell lines. Univariate survival analysis was conducted according to the Kaplan-Meier method, and the difference between the survival curves was analyzed with the log-rank test. Multivariate survival analysis was performed using the Cox proportional hazard model. A positive coefficient indicates a worse prognosis, and a negative coefficient indicates a protective effect of the variable with which it is associated. Hazard ratios (HRs) and 95\% confidence intervals (CIs) were obtained to assess the prognostic effects of each variable on the outcomes. Values of $\mathrm{P}<0.05$ were considered statistically significant.

\section{Results}

The level of RNF43 was significantly down-regulated in gastric carcinoma

The RNF43 mRNA and protein in the gastric carcinoma cell lines and the gastric epithelial cell line were respectively assessed by q-PCR and western blotting. As shown in Fig. $1 \mathrm{a}$ and $\mathrm{b}$, the RNF43 mRNA and protein expressions were significantly lower in the gastric carcinoma cell lines (BGC823, 7901, and MGC803) than in the normal epithelial cell line (GES-1; all $\mathrm{P}<0.005$ ). The same results were also confirmed in 4 cases of gastric carcinomas and matched normal mucosal tissues (Fig. $1 \mathrm{c}, \mathrm{d}$ ).

\section{Clinicopathological characteristics of the patients}

The ages of the 77 patients ranged from 24 to 86 years (median 65 years; mean 59.6 years). Among these patients, the pathological diagnoses were well-differentiated adenocarcinoma in 4 cases, moderately differentiated adenocarcinoma in 14 cases, and poorly differentiated adenocarcinoma in 59 cases. According to the pathological TNM stage, 16 cases were stage I, 30 were stage II, 31 were stage III, and 0 were stage IV. During the follow-up period, 54 patients died, and 23 were alive at the end of follow up median survival time 44 months, mean survival time $46.4 \pm 3.3$ months).

The expression of RNF43 was correlated to patient's clinicopathological features and survival

We detected the lack of expression of RNF43 in the tumor tissues more frequently than that in the normal mucosal tissues $(\mathrm{P}<0.001)$. RNF43 expression was observed in the cytoplasm (Fig. 2). RNF43 expression was lower in the larger tumors ( $<<0.001$ ). A lack of expression of RNF43 was not found in the well-differentiated adenocarcinomas, in $31.25 \%$ ( 5 out of 16 ) of the moderately differentiated adenocarcinomas, and in $72.22 \%$ (39 out of 54) of the poorly differentiated adenocarcinomas. RNF43 expression was positively correlated with histological differentiation $(\mathrm{P}=0.001)$. RNF43 expression was lower in advanced T-stage cancer $(\mathrm{P}<0.001)$. RNF43 expression was found to be positively correlated with depth of invasion and metastases of the regional lymph nodes $(\mathrm{P}<0.001)$. An apparent 
Fig. 1. a: The RNF43 mRNA expression level in a gastric epithelial cell line (GES-1) and gastric carcinoma cell lines (MGC803, BGC823 and SGC7901) was analyzed by quantitative reverse transcription polymerase chain reaction (qRT-PCR), and was normalized to that of glyceraldehyde 3-phosphate dehydrogenase (GAPDH). The mean (SD) mRNA expression levels for RNF43 were approximately 0.224 -fold, 0.404 fold, and 0.269-fold in the MGC803, BGC823 and SGC7901 cell lines, respectively, as compared with the expression in GES-1 cells $\left({ }^{*} \mathrm{P}<0.001\right)$. b: The expression of RNF43 protein was analyzed by Western blot. RNF43 expression was significantly lower in the MGC803, BGC823 and SGC7901 cell line than in GES-1 cells. GAPDH was used as loading control. c: The RNF43 mRNA expression level in 4 cases of gastric carcinoma tissue and matched gastric normal tissue was analyzed by qRT-PCR and was normalized to that of GAPDH. The mean (SD) mRNA expression levels for RNF43 were approximately 0.387 -fold, 0.207-fold, 0.174-fold and0.191-fold in gastric carcinoma tissue compared with the expression in gastric normal tissue $\left({ }^{*} \mathrm{P}<0.001\right) . \mathrm{d}$ : The expression of RNF43 protein was analyzed by Western blot. RNF43 expression was significantly lower in gastric carcinoma tissue than in gastric normal tissue. GAPDH was used as loading control. a

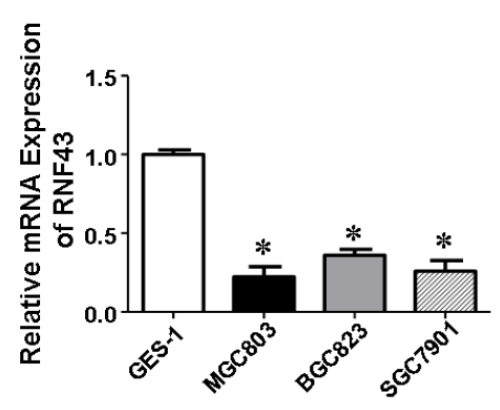

b

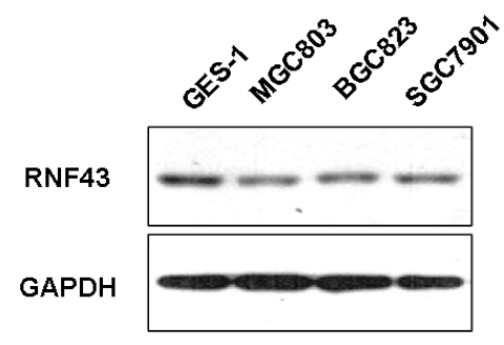

C

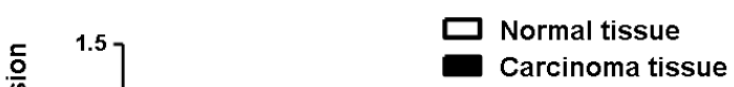

d

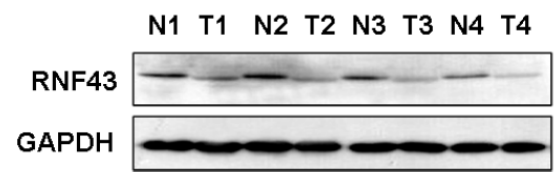

relationship was found between the frequency of RNF43 expression and the pTNM stage, and observations of the lack of expression of RNF43 gradually increased with the progression of pTNM stage $(\mathrm{P}<0.001$; Table 1$)$.

Survival curves were calculated according to Kaplan-Meier analysis, and the survival analysis with the log-rank test suggested that RNF43-negative patients exhibited a significantly shorter survival time (median 35 months, mean 37.78.53 \pm 4.34 months) than did the RNF43-positive patients (median 85 months, mean $67.52 \pm 5.32$ months) (log-rank test, $\mathrm{P}<0.001$ ) (Fig. 3). The 5-year survival rates were $18.2 \%$ for the RNF43-negative patients and $60.6 \%$ for the RNF43-positive patients. Multivariate analysis was performed using the Cox regression. RNF43 was found to significantly affect the outcome of gastric cancer and appeared to be an independent prognostic factor $(\mathrm{P}=0.021)$ with a hazard ratio (HR) of 0.24 (95\% confidence interval [CI] 0.072-0.804). Histological differentiation (HR 2.318, 95\% CI 1.081-4.971, $\mathrm{P}=0.031$ ), the depth of invasion (HR 3.565, 95\% CI 1.702-7.471, P =0.001), and lymph node metastasis (HR 2.201, 95\% CI 1.207-4.013, P =0.01; Table 2) also affected the outcomes. 
Fig. 2. Immunohistochemical analysis of RNF43 in gastric carcinoma and adjacent normal tissues. Staining of RNF43 in adjacent normal tissue (strong positive, $a \times 100, b \times 200$ ), highly differentiated gastric carcinoma tissue (moderately positive, c $\times 200, d \times 400$ ), c moderately differentiated gastric carcinoma tissue (weakly positive, e $\times 200, f \times 400$ ) . poorly differentiated gastric carcinoma tissue (negative, $\mathrm{g} \times 100$, $\mathrm{h} \times 200$ ) .
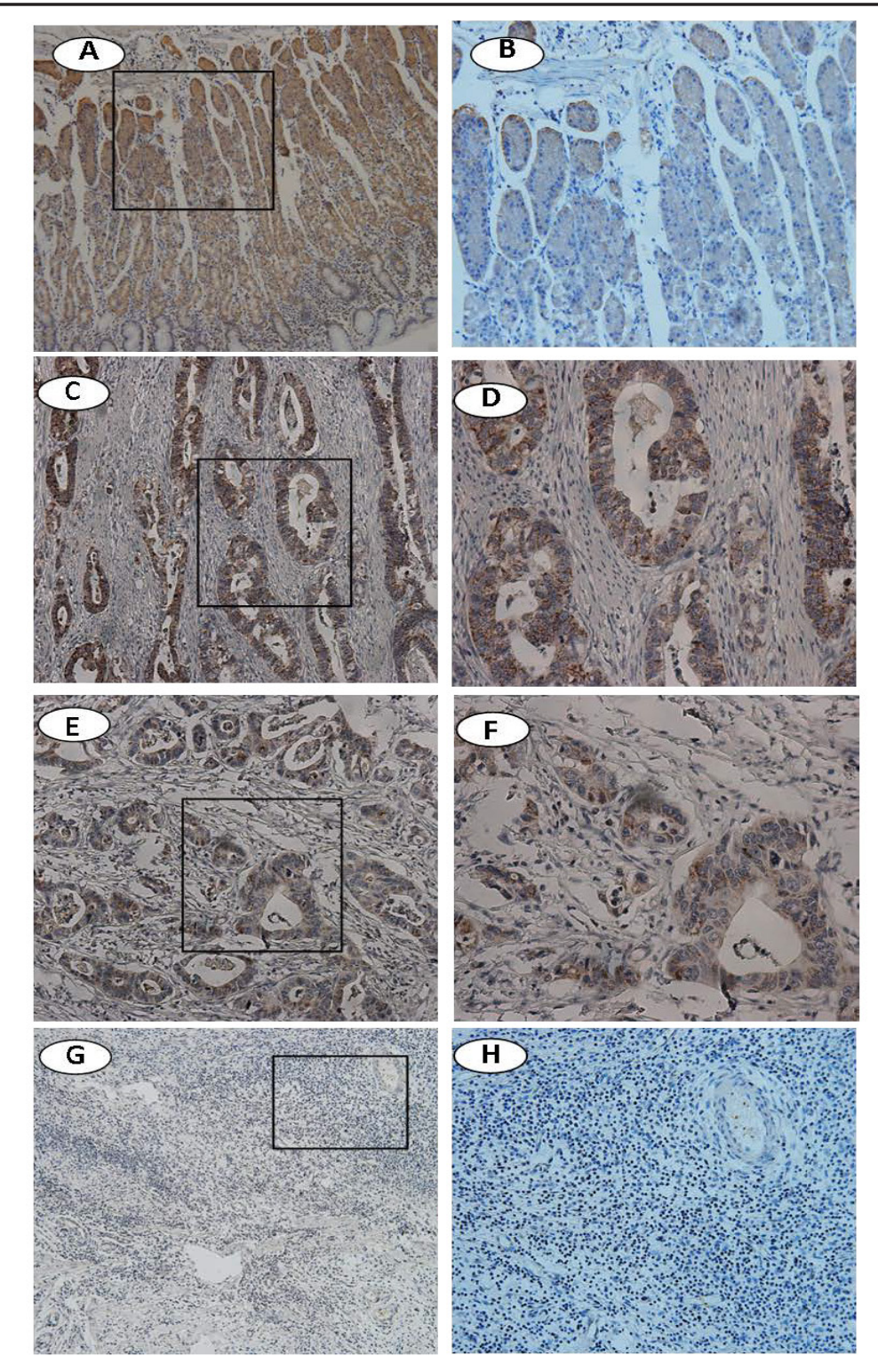

Fig. 3. Kaplan-Meier survival curves for the 77 gastric carcinoma patients, based on RNF43 expression. The $y$ axis represents the percentage of patients, and the $\mathrm{x}$ axis represents their survival in months. RNF43-positive patients showed significantly longer survival than RNF43-negative patients by log-rank test $(\mathrm{P}<0.001)$. cum cumulative.

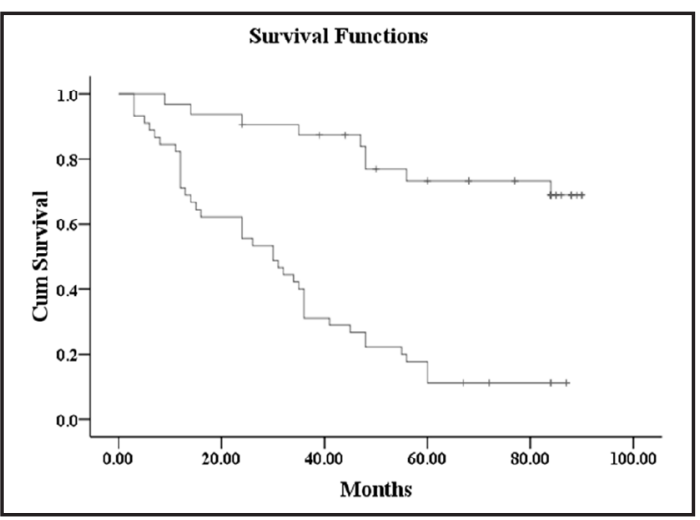

RNF43 inhibits cell proliferation but promotes cell apoptosis

MGC803 cells and GES-1 cells were infected with RNF43 recombinant adenovirus. The cell growth curves revealed that the over-expression of RNF43 significantly suppressed cell 
Table 1. Correlation between RNF43 expression and clinic-pathological variables in gastric carcinoma. $\mathrm{P}<0.05$, statistically significant

\begin{tabular}{|c|c|c|c|}
\hline \multirow[t]{2}{*}{ Variable } & \multicolumn{2}{|l|}{ RNF43 } & \multirow[t]{2}{*}{$P$ value } \\
\hline & Positive (\%) & Negative (\%) & \\
\hline \multicolumn{4}{|l|}{ Gender } \\
\hline Male & 24 & 34 & 0.56 \\
\hline Female & 9 & 10 & \\
\hline \multicolumn{4}{|l|}{ Age } \\
\hline$n<45$ years & 2 & 4 & 0.904 \\
\hline$n=45-60$ years & 21 & 24 & \\
\hline$n \geq 60$ years & 10 & 16 & \\
\hline \multicolumn{4}{|l|}{ Tumor size (diameter; d) } \\
\hline $\mathrm{d}<4 \mathrm{~cm}$ & 11 & 5 & $<0.001$ \\
\hline $\mathrm{d}=4-8 \mathrm{~cm}$ & 22 & 28 & \\
\hline$d \geq 8 \mathrm{~cm}$ & 0 & 9 & \\
\hline \multicolumn{4}{|l|}{ Histological differentiation } \\
\hline Well-differentiated & 7 & 0 & 0.001 \\
\hline Moderately differentiated & 11 & 5 & \\
\hline Poorly differentiated & 15 & 39 & \\
\hline \multicolumn{4}{|l|}{ Depth of invasion } \\
\hline T1 & 0 & 0 & $<0.001$ \\
\hline $\mathrm{T} 2$ & 26 & 8 & \\
\hline T3 & 7 & 29 & \\
\hline $\mathrm{T} 4$ & 0 & 7 & \\
\hline \multicolumn{4}{|l|}{ Lymph node metastasis } \\
\hline No & 22 & 8 & $<0.001$ \\
\hline N1 & 9 & 8 & \\
\hline N2 & 1 & 11 & \\
\hline N3 & 1 & 18 & \\
\hline \multicolumn{4}{|l|}{ pTNM stage } \\
\hline I & 16 & 0 & $<0.001$ \\
\hline II & 16 & 15 & \\
\hline III & 1 & 29 & \\
\hline IV & 0 & 0 & \\
\hline
\end{tabular}

Table 2. Cox regression analysis of prognostic factors in gastric carcinoma

\begin{tabular}{|c|c|c|c|c|c|c|c|}
\hline \multirow[t]{2}{*}{ Prognostic variables } & \multirow[t]{2}{*}{ B } & \multirow[t]{2}{*}{ SE } & \multirow[t]{2}{*}{ Wald value } & \multirow[t]{2}{*}{$P$ value } & \multirow[t]{2}{*}{ HR } & \multicolumn{2}{|c|}{ 95\% CI for HR } \\
\hline & & & & & & Lower & Upper \\
\hline Gender & 0.1 & 0.353 & 0.081 & 0.776 & 1.106 & 0.554 & 2.207 \\
\hline Age & 0.368 & 0.294 & 1.561 & 0.211 & 1.444 & 0.811 & 2.57 \\
\hline Size & 0.515 & 0.359 & 2.058 & 0.151 & 1.674 & 0.828 & 3.385 \\
\hline Histological differentiation & 0.841 & 0.389 & 4.662 & 0.031 & 2.318 & 1.081 & 4.971 \\
\hline Depth of invasion & 1.271 & 0.377 & 11.345 & 0.001 & 3.565 & 1.702 & 7.471 \\
\hline RNF43 & -1.428 & 0.617 & 5.349 & 0.021 & 0.24 & 0.072 & 0.804 \\
\hline
\end{tabular}

proliferation compared to the negative control group in a time-dependent manner $(\mathrm{P}<0.05$, Fig. 4a). Seventy-two hours after infection, cell apoptosis was determined by FACS. As shown in Fig. 4c, compared to the negative control groups, significant increases in cell apoptosis were observed in the MGC803 cells and GES-1 cells infected with adenovirus encoding RNF43 gene ( $\mathrm{P}<0.05$, Fig. 4e). Furthermore, BGC823 cells and GES- 1 cells were infected with lentivirus harboring RNF43 siRNA. The cell growth curves revealed that the knockdown of RNF43 significantly promoted cell proliferation compared to the negative control group in a time-dependent manner $(\mathrm{P}<0.05$, Fig. $4 \mathrm{~b})$. Seventy-two hours after infection, cell apoptosis was determined by FACS. As shown in Fig. 4d, compared to the negative control group, a trend 


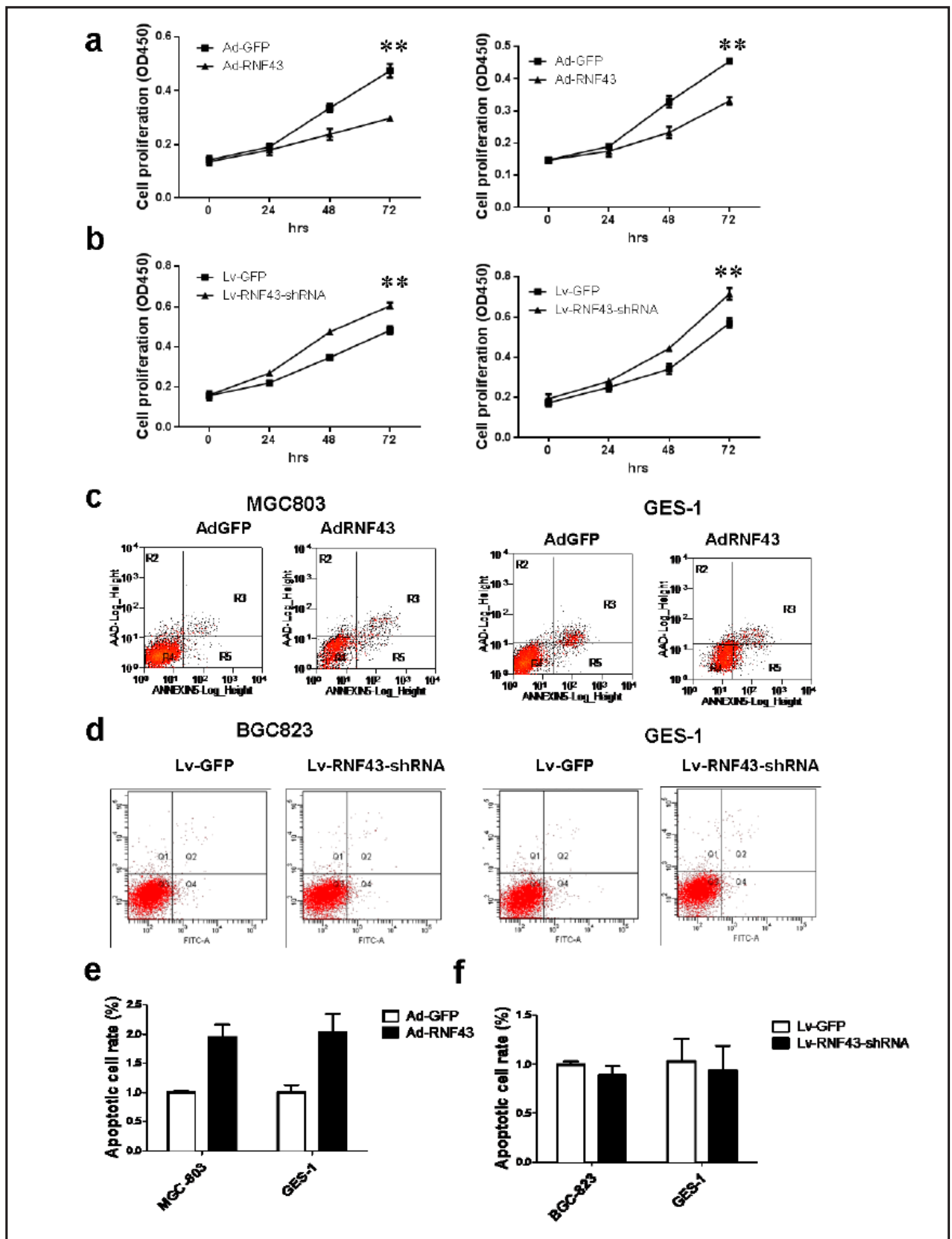

Fig. 4. Effects of RNF43 on proliferation and apoptosis by MGC803, BGC823 and GES-1cells. Over-expression and knockdown of RNF43 was achieved by infecting AdRNF43 and Lv-RNF43-shRNA. All cell lines infected with negative control as control. CCK-8 assay was performed to measure cell proliferation at the indicated time ( $\mathrm{a}$ is result of over- expression, $\mathrm{b}$ is result of knockdown). Cell apoptosis was evaluated by using Annexin V/propidium iodide-double staining. Early and late apoptotic cells were combined as Annexin V-positive cells that were employed as the criterion to calculate the percentage of cell apoptosis(c is result of over- expression, $d$ is result of knockdown). Bar charts shown are the representative set of two independent experiments, and are mean \pm SD compared with the negative control (e is result of over- expression, ${ }^{*} \mathrm{P}<0.05$, $\mathrm{f}$ is result of knockdown, $\mathrm{P}>0.05$ ). 
Fig. 5. MGC803 and GES-1 cells were infected with Ad-RNF43 as the over-expression group, with AdGFP as negative control. Over-regulation of p53 and cleaved-caspase 3 and down-regulation of Ki67 and Lgr5 both in MGC803 and GES- 1 cells by Ad-RNF43 was determined by Western blot (a). BGC823 and GES-1 cells were infected with Lv-RNF43-shRNA as the knockdown group, with Lv-GFP as negative control. Down-regulation of p53 and cleaved-caspase 3 and over-regulation of Ki67 and Lgr5 both in BGC823 and GES-1 cells by Lv-RNF43-shRNA was determined by Western blot (b).

toward the suppression of cell apoptosis was observed in the BGC823 cells and GES-1cells after knockdown of RNF43 (P>0.05, Fig. 4f). These results further support the potential anticancer activity of RNF43 in gastric carcinoma.

RNF43 positive correlated to p53 and cleaved-caspase3 but negative related to Ki67and Lgr5

MGC803 cells and GES-1 cells were infected with recombinant adenovirus encoding RNF43 or encoding EGFP as negative controls. Western blot tests revealed that the over-expression of RNF43 significantly upgulated the expression levels of p53 and cleaved-caspase3 in the MGC803 and GES- 1 cell lines compared to the negative control groups (Fig. 5a). An interference test

a

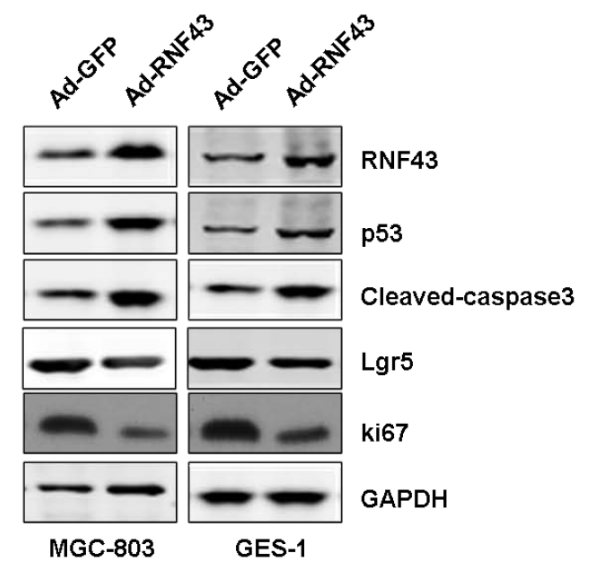

b
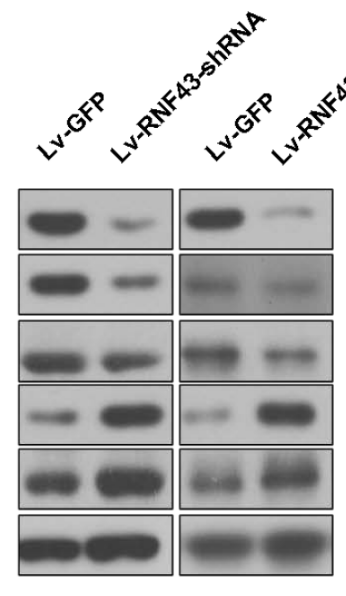

RNF43

p53

Cleaved-caspase 3

Lgr5

ki67

GAPDH

BGC-823 GES-1

with shRNF43 revealed that the reduction of RNF43 resulted in significant decreases in the expressions of p53 and cleaved-caspase3 in the BGC823 and GES-1 cell lines (Fig. 5b). Additionally, we observed that the expressions of Ki67 and Lgr5 were inversely regulated by RNF43 in both the over-expression and interference tests (Fig. 5). These results demonstrate that RNF43 might have functions similar to those of p53 or cleaved-caspase 3 and was inversely related to Ki67 and Lgr5.

\section{Discussion}

Ring finger protein 43 (RNF43) belongs to the family of E3 ubiquitin-protein ligases. Previous studies have identified RNF43 as a novel oncoprotein in colorectal cancer [1719]. The current study demonstrated that RNF43 acts as an inhibitor of Wnt signaling by negatively regulating frizzled receptor surface expression [9]. Scholars also found that RNF43 is a tumor suppressor gene in mucinous ovarian tumors, and similar findings have been reported in studies of mucinous pancreatic precancerous cysts [20-22]. However, the function of RNF43 in human gastric carcinoma has never been reported.

In our study, we focused our attention on examining the expression level and function of RNF43 in gastric carcinomas. We hypothesized that RNF43 might also act as an inhibitor in gastric carcinoma cell proliferation as it has been reported to do in other types of cancer. Therefore, we investigated the mRNA and protein levels of RNF43 in three gastric carcinoma 
cell lines and one gastric epithelial cell line with qPCR and western blot analyses. Both test methods revealed that the expressions of RNF43 mRNA and protein in the gastric carcinoma cell lines were significantly down-regulated compared to those in the gastric epithelial cell line. We also investigated the above indicators in four pairs of gastric carcinoma tissues and found that the results were similar to those from the cell lines.

Our study investigated RNF43 in a certain number of gastric carcinoma samples with available follow-up data and showed that RNF43 expression was significantly reduced in the gastric carcinoma tissues compared to the distal normal mucosae. The decreased expression of RNF43 was significantly correlated with histological differentiation, tumor size, depth of invasion, lymph node metastasis, pTNM stage and survival. These results suggest that low expression levels of RNF43 are typically correlated with more malignant and metastatic tumors. RNF43 expression was found to be absent in $48.39 \%(15 / 31)$ of the stage II cancer tissues and $96.67 \%$ (29/30) of the stage III cancer tissues. We also found that low expression levels of RNF43 were closely related to the worst prognoses for gastric carcinoma. These results suggest that RNF43 might play a suppressive role in the development and progression of these tumors. Because the combination therapy of peptide vaccines and anti-cancer drugs for colorectal cancer is associated with increased expression of RNF43 [23, 24], a novel therapeutic strategy based on RNF43 might be used in the future for patients with gastric carcinomas. Additionally, we analyzed the reason that we observed that there was no statistical association between pTNM and survival in the Cox proportional hazard model and found that the number of samples might have been insufficient.

In the in vitro experiments, we confirmed the anti-tumor effect of RNF43 in gastric carcinoma. Our results revealed that RNF43 had large and obvious effects on the proliferation and apoptosis of MGC803, BGC823 and GES-1 cells. These results revealed that, among three gastric carcinoma cell lines, the expression of RNF43 was highest in the BGC823 cells and lowest in the MGC803 cells. Therefore, we chose to over-express RNF43 in the MGC803 cell line with the pEGFP-RNF43 recombinant adenovirus and the BGC823 cell line to knock down RNF43 with the lentivirus shRNF43. The two methods were both performed in the normal epithelial cell line (GES-1). The CCk-8 and flow cytometric assays revealed that infection with pEGFP-RNF43 recombinant adenovirus significantly inhibited proliferation and promoted apoptosis in the MGC803 and GES-1 cells. In contrast, infection with shRNF43 significantly promoted the proliferation of BGC823 and GES-1 cells and limited the inhibition of apoptosis. We suggest that low levels of the expression of RNF43 might not inhibit the apoptosis but promote the proliferation of both carcinoma and normal cells. The classical tumor suppressor gene p53 is the gene that is most frequently mutated and inactivated [25, 26]. Caspase 3 is a type of photolytic enzyme that mediates cell apoptosis [27]. In our study, we observed positive relationships between RNF43, p53, and cleaved-caspase3. These data suggest that low levels of the expression of RNF43 might decrease apoptosis and promote proliferation of gastric carcinoma cells and might be one of conditions which result of the conversion to normal gastric epithelial cells to cancer. These data also suggest that RNF43 might act as a tumor suppressor in gastric carcinoma.

The leucine-rich repeat-containing G protein-coupled receptor Lgr5 has been identified as a novel stem cell marker in the intestinal epithelia, stomach, and hair follicles [28-30]. It has been confirmed RNF43 is specifically expressed in Lgr5-positive small intestinal crypt cell and inhibits cell proliferation. Researchers have found that Lgr5 participates in the degradation of rnf43/znrf3. Recently, scholars have analyzed the protein complex domain of Rspod1-Lgr5-rnf43/znrf3 and suggested thatLgr5 participates in the degradation of RNF43/ZNRF3 in vitro [31, 32]. We conducted a test to determine whether RNF43 and Lgr5 exert similar effects in gastric carcinomas. We detected the Lgr5 protein levels following pEGFP-RNF43 recombinant adenovirus infection in both the gastric carcinoma cell line and the gastric epithelial cell line. Compared to the negative controls, the over-expression of RNF43 led to decreased expression of Lgr5. A similar result was also found in the interference test with shRNF43.These results indicate that RNF43 might be inversely related to the potential of the cancer stem cell marker Lgr5. The elucidation of the exact mechanism

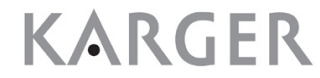


will require further effort in future studies. Our study also revealed that RNF43 expression was negatively correlated with Ki-67, which is recognized nuclear antigen-specific marker of cellular proliferation; this finding suggests that RNF43-negative cancer cells have higher proliferative activity.

In conclusion, our study used immunohistochemical methods to reveal that the expression of RNF43 was significantly lower in gastric cancer tissue than in normal mucosal tissue. This result was confirmed by western blot analysis. Furthermore, low levels of the expression of RNF43 in primary gastric carcinoma were significantly correlated with shorter survival, and low RNF43 expression was a significant predictor of poorer survival. RNF43 expression was also associated with gastric carcinoma cell proliferation. We believe that these findings can help to predicting clinical outcomes and contribute to the understanding of the inhibition of gastric carcinoma. Further investigations of RNF43 are required to identify its mechanism of action in gastric carcinoma and to develop new therapeutic agents.

\section{Acknowledgements}

We thank Professor Lin-Xin Wei and Dr. Jing Yuan, Department of Pathology, Chinese People's Liberation Army General Hospital, for their excellent technical assistance. This work was supported by a National Natural Science Foundation of China (Grant No. 81272698).

\section{Disclosure Statement}

We declare that we have no conflict of interest.

\section{References}

1 Thun MJ, DeLancey JO, Center MM, Jemal A, Ward EM: The global burden of cancer: priorities for prevention. Carcinogenesis 2010;31:100-110.

-2 Xu XY, Zhang LJ, Yu YQ Zhang XT, Huang WJ, Nie XC, Song GQ: Down-regulated MAC30 expression inhibits proliferation and mobility of human gastric cancer cells. Cell Physiol Biochem 2014;33:1359-1368.

3 Chen X, Leung SY, Yuen ST, Chu KM, Ji J, Li R, Chan AS, Law S, Troyanskaya OG, Wong J, So S, Botstein D, Brown PO: Variation in gene expression patterns in human gastric cancers. Mol Biol Cell 2003;14:32083215.

4 Freese JL, Pino D, Pleasure SJ: Wnt signaling in development and disease. Neurobiol Dis 2010;38:148-153.

5 Cui Y, Chen J, He Z, Xiao Y: SUZ12 depletion suppresses the proliferation of gastric cancer cells. Cell Physiol Biochem 2013;31:778-784.

6 Clevers H: Wnt/ $\beta$-catenin signaling in development and disease. Cell 2006;127:469-480.

7 Anastas JN, Moon RT: WNT signalling pathways as therapeutic targets in cancer. Nat Rev Cancer 2013;13:11-26.

8 Hao H-X, Xie Y, Zhang Y, Charlat O, Oster E, Avello M, Lei H, Mickanin C, Liu D, Ruffner H, Mao X, Ma Q, Zamponi R, Bouwmeester T, Finan PM, Kirschner MW, Porter JA, Serluca FC, Cong F: ZNRF3 promotes Wnt receptor turnover in an R-spondin-sensitive manner. Nature 2012;485:195-200.

-9 Koo B-K, Spit M, Jordens I, Low TY, Stange DE, van de Wetering M, van Es JH, Mohammed S, Heck AJ, Maurice MM, Clevers H: Tumour suppressor RNF43 is a stem-cell E3 ligase that induces endocytosis of Wnt receptors. Nature 2012;488:665-669.

10 MacDonald BT, He X: A finger on the pulse of Wnt receptor signaling. Cell Res 2012;22: 1410-1412.

11 Livak KJ, Schmittgen TD: Analysis of relative gene expression data using real-time quantitative PCR and the 2(-Delta Delta C(T)) Method. Methods (San Diego, Calif) 2001;25:402-408.

12 Zhou X, Xia Y, Su J, Zhang G: Down-regulation of miR-141 induced by helicobacter pylori promotes the invasion of gastric cancer by targeting STAT4. Cell Physiol Biochem 2014;33:1003-1012. 


\section{Cellular Physiology Cell Physiol Biochem 2015;36:1835-1846 \begin{tabular}{l|l|l} 
and Biochemistry & $\begin{array}{l}\text { DOI: 10.1159/000430154 } \\
\text { Publisned onlne: July 13, } 2015\end{array}$ & $\begin{array}{l}\text { O) 2015 S. Karger AG, Basel } \\
\text { www.karger.com/cpb }\end{array}$ \\
\cline { 2 - 3 }
\end{tabular}}

Niu et al.: RNF43 Might Act as a Tumor Suppressor in Gastric Carcinoma

13 Xi H-Q Zhao P, Han W-D: Clinicopathological significance and prognostic value of LRP16 expression in colorectal carcinoma. World journal of gastroenterology: WJG 2010;16:1644.

14 Matsubara J, Yamada Y, Nakajima TE, Kato K, Hamaguchi T, Shirao K, Shimada Y, Shimoda T: Clinical significance of insulin-like growth factor type 1 receptor and epidermal growth factor receptor in patients with advanced gastric cancer. Oncology 2007;74:76-83.

15 Wood LD, Calhoun ES, Silliman N, Ptak J, Szabo S, Powell SM, Riggins GJ, Wang TL, Yan H, Gazdar A, Kern SE, Pennacchio L, Kinzler KW, Vogelstein B, Velculescu VE: Somatic mutations of GUCY2F, EPHA3, and NTRK3 in human cancers. Hum Mutat 2006;27:1060-1061.

16 Xi H-Q Zhao P: Clinicopathological significance and prognostic value of EphA3 and CD133 expression in colorectal carcinoma. J Clin Pathol 2011;64:498-503.

17 Uchida N, Tsunoda T, Wada S, Furukawa Y, Nakamura Y, Tahara H: Ring finger protein 43 as a new target for cancer immunotherapy. Clin Cancer Res 2004;10:8577-8586.

18 Yagyu R, Furukawa Y, Lin Y-M, Shimokawa T, Yamamura T, Nakamura Y: A novel oncoprotein RNF43 functions in an autocrine manner in colorectal cancer. Int J Oncol 2004;25:1343-1348.

19 Sun Y: E3 ubiquitin ligases as cancer targets and biomarkers. Neoplasia 2006;8:645-654.

20 Ryland GL, Hunter SM, Doyle MA, Rowley SM, Christie M, Allan PE, Bowtell DD, Gorringe KL, Campbell IG: RNF43 is a tumour suppressor gene mutated in mucinous tumours of the ovary. J Pathol 2013;229:469476.

-21 Zou Y, Wang F, Liu F-Y, Huang MZ, Li W, Yuan XQ Huang OP, He M: RNF43 mutations are recurrent in Chinese patients with mucinous ovarian carcinoma but absent in other subtypes of ovarian cancer. Gene 2013;531:112-116.

22 Jiang X, Hao H-X, Growney JD, Woolfenden S, Bottiglio C, Ng N, Lu B, Hsieh MH, Bagdasarian L, Meyer R, Smith TR, Avello M, Charlat O, Xie Y, Porter JA, Pan S, Liu J, McLaughlin ME, Cong F: Inactivating mutations of RNF43 confer Wnt dependency in pancreatic ductal adenocarcinoma. P Natl A Sci 2013;110:1264912654.

-23 Yoshimatsu K, Yokomizo H, Osawa G, Fujimoto T, Otani T, Tsunoda T, Nakamura Y, Ogawa K: Phase I study of combination therapy with peptide vaccine and anti-cancer drug for colorectal cancer. Gan to kagaku ryoho Cancer \& chemotherapy 2008;35:2268-2270.

24 Okuno K, Sugiura F, Hida JI, Tokoro T, Ishimaru E, Sukegawa Y, Ueda K: Phase I clinical trial of a novel peptide vaccine in combination with UFT/LV for metastatic colorectal cancer. Exp Ther Med 2011;2:73-79.

25 Tewari M, Krishnamurthy A, Shukla HS: Predictive markers of response to neoadjuvant chemotherapy in breast cancer. Surg Oncol 2008;17:301-311.

26 Cho Y, Gorina S, Jeffrey PD, Pavletich NP: Crystal structure of a p53 tumor suppressor-DNA complex: understanding tumorigenic mutations. Science 1994;265:346-355.

27 Porter AG, Janicke RU: Emerging roles of caspase-3 in apoptosis. Cell Death Differ 1999;6:99-104.

28 Barker N, Clevers H: Tracking down the stem cells of the intestine: strategies to identify adult stem cells. Gastroenterology 2007;133:1755-1760.

29 Barker N, Huch M, Kujala P, van de Wetering M, Snippert HJ, van Es JH, Sato T, Stange DE, Begthel H, van den Born M, Danenberg E, van den Brink S, Korving J, Abo A, Peters PJ, Wright N, Poulsom R, Clevers H: Lgr5(+ve) stem cells drive self-renewal in the stomach and build long-lived gastric units in vitro. Cell stem cell 2010;6:25-36.

-30 Jaks V, Barker N, Kasper M, van Es J. H, Snippert HJ, Clevers H, Toftgard R: Lgr5 marks cycling, yet longlived, hair follicle stem cells. Nat Genet 2008;40:1291-1299.

-31 de Lau W, Peng WC, Gros P, Clevers H: The R-spondin/Lgr5/Rnf43 module: regulator of Wnt signal strength. Genes Dev 2014;28:305-316.

32 Zebisch M, Xu Y, Krastev C, MacDonald BT, Chen M, Gilbert R. J, He X, Jones EY: Structural and molecular basis of ZNRF3/RNF43 transmembrane ubiquitin ligase inhibition by the Wnt agonist R-spondin. Nat Commun 2013;4:2787. 